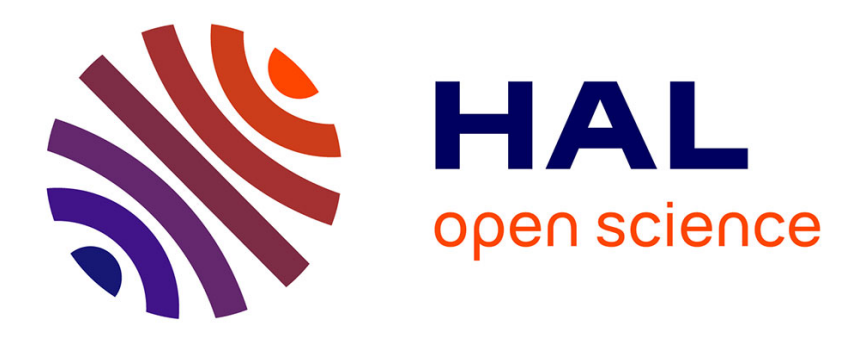

\title{
Dynamic grouping maintenance policy for the road infrastructure
}

Ikram Najeh, Laurent Bouillaut, Dimitri Daucher, Maxime Redondin

\section{To cite this version:}

Ikram Najeh, Laurent Bouillaut, Dimitri Daucher, Maxime Redondin. Dynamic grouping maintenance policy for the road infrastructure. ESREL2021, 31st European Safety and Reliability Conference, Sep 2021, Angers, France. 7p. hal-03367284

\section{HAL Id: hal-03367284 \\ https://hal.science/hal-03367284}

Submitted on 8 Nov 2021

HAL is a multi-disciplinary open access archive for the deposit and dissemination of scientific research documents, whether they are published or not. The documents may come from teaching and research institutions in France or abroad, or from public or private research centers.
L'archive ouverte pluridisciplinaire HAL, est destinée au dépôt et à la diffusion de documents scientifiques de niveau recherche, publiés ou non, émanant des établissements d'enseignement et de recherche français ou étrangers, des laboratoires publics ou privés. 


\title{
Dynamic grouping maintenance policy for the road infrastructure
}

\author{
Ikram Najeh \\ Vedecom, 23 Bis Allée des Marronniers, 78000 Versailles, France. E-mail: $\underline{\text { ikram.najeh@vedecom.fr }}$
}

Laurent Bouillaut

Université Gustave Eiffel, 5 Boulevard Descartes, 77420 Champs-sur-Marne, France.E-mail: laurent.bouillaut@univ-eiffel.fr

Dimitri Daucher

Université Gustave Eiffel, 5 Boulevard Descartes, 77420 Champs-sur-Marne, France.E-mail: dimitri.daucher@univ-eiffel.fr

Maxime Redondin

Vedecom, 23 Bis Allée des Marronniers, 78000 Versailles, France.E-mail: ikram.najeh@vedecom.fr

\begin{abstract}
The quality and the ability of the road infrastructures play a very important role to ensure safe and convenient transportation. Current human needs oblige us to optimize the use of our travel resources. The autonomous driving is proposed as a solution to make the transport more efficient. The autonomous vehicle contains cameras and lidars that can communicate with the road infrastructure to understand the environment where they are traveling. Thus, the existing road infrastructure must therefore be developed and maintained to address the availability needs induced by the new uses of mobility. In consequence, the maintenance of the road infrastructure must be optimized.

In previous work, a maintenance policy for lines and road cracks pavement of the road infrastructure was proposed by considering it as a 4 components system: median strip line, emergency line, broken center line and pavement. The proposed strategy is based on the individual optimal maintenance plan of each component. Then, over a finite planning horizon, the scheduled maintenance actions are grouped together to ensure both the proper functioning of the system and to minimize the cost of maintenance.

To improve this previous work, a new long-term horizon (30 years) dynamic grouping maintenance strategy is proposed, dynamically considering new monitoring data. This new algorithm is applied to the maintenance optimization of the road infrastructure, using the Long-Term Pavement Performance database and French National Road 4 feedback data.
\end{abstract}

Keywords: Grouping maintenance, Dynamic grouping, Road infrastructure, Genetic Algorithm, Multi-component system, Preventive maintenance, Autonomous vehicle

\section{Introduction}

Autonomous vehicles are becoming a new piece of infrastructure. This type of vehicles contains cameras and sensors able to communicate with the road infrastructure to understand the environment where they are traveling. The information given by the road infrastructure are used by the vehicle to generate situational awareness of the car and its surroundings and make appropriate decision while driving.

Thus, an efficient maintenance for the road infrastructure is necessary. The various defects of the road infrastructure (whether it is the pavement or the road markings) are incompatible phenomena with the operation of this type of vehicle. These defects are often in the form of cracks for the pavement (longitudinal, transversal...) or in the form marking degradation (loss of retro reflection). An example showing the need for preventive and efficient maintenance for the infrastructure to accommodate the autonomous vehicle on the existing infrastructure is given in Revilloud et al 2016. In some instances, the road marking is not detected by the camera of the vehicle. This is sometimes due to the appearance of cracks on the road markings. Thus, an efficient maintenance for the infrastructure must be considered.

I. Najeh et al (2020) prosed a grouping maintenance strategy for the road infrastructure. It considered the road infrastructure as serial system with 4 components: 3 road lines (in some cases only 2 marking lines exist) and the pavement. It was based on the feedback data inspection of the French RN4 marking lines and the pavement data provided from the American database "Long Term Pavement Performance (LTPP)" (limited to Texas). Each component has its own maintenance strategy and using Genetic Algorithm the optimal grouping solution is defined.

During the last years, maintenance strategies for multicomponent systems was discussed by many authors. Varied papers take advantages of positive economic dependences 
between the system's component to propose a grouping maintenance strategy. It implies that combining maintenance activities is cheaper than performing maintenance on components separately. These strategies are often applied in case of serial systems or systems with complex structure to take advantages of downtime of a component to maintain another. The nature of the planning horizon determines the maintenance grouping policy: infinite horizon (stationary policy) and finite horizon (dynamic policy). According to K. Bouvard et al (2011) stationary models assume that the system evolves during a long-term stable operating environment. Thus, maintenance plans are generated consistent with static rules. That means that each maintenance dates are scheduled at regular time intervals. In the other hand, dynamic models can incorporate dynamically short-term information just like the varying use of components, unexpected component failure or the change of maintenance costs. They supply dynamic maintenance dates which change over the design horizon. In this paper, the dynamic grouping maintenance strategy is considered.

P. Do Van et al (2013) proposed dynamic grouping maintenance strategy with time limited opportunities for multi-component systems with positive economic dependence. It based on taken advantages of inactivity period (called maintenance opportunities) to perform a preventive maintenance. The proposed strategy is economically powerful when short-term information becomes available. R.E Wildeman et al (1997) proposed a dynamic grouping policy for maintenance activities by suggesting a fast approach that allows interactive planning by showing how shifts from the tentative planning work out. M. Sheikhalishahi et al (2017) proposed a dynamic planning process taking into consideration grouping strategy in addition to human factors. However, the impact of human factors is different in industry. This strategy minimizes the maintenance cost while human error as well as risks of delaying maintenance activities are taken into account.

\section{Feedback data}

In the lake of a "complete" database that contains both feedback data inspection of the pavement and road marking, the proposed strategy is applied on the feedback data of the road marking of the French National Road 4 (NR 4) (Median strip line, Emergency line and Broken centre line) and the pavement data of the "Long Term Pavement Performance" (LTPP) database.

The road markings of (NR4) have the particularity being retroreflective. Retroreflectivity designs the ability of a surface to return light to its source. In this paper, a road marking is considered as a "efficient" one if its retroreflection level is a least $150 \mathrm{mcd} / \mathrm{m}^{2} / \mathrm{lx}$. The road markings are inspected once a year.

For the pavement, an American database (LTPP) is used. It is a program founded by the Federal HighWay
Administration (FHWA) since 1988. It collects the data performance the American pavement in different states. In this work, the Texas data is therefore considered. The percent of longitudinal and fatigue cracks is the decision parameter. The Texas sections are inspected one two years and it considered failed if the cracks percent is greater than $5 \%$.

As mentioned in the previous paragraph, the road infrastructure components are periodically inspected. Thus, the exact time of failure is not observed. In this case, the data is censored.

To simplify the study, the point repair of the road markings and the pavement sections are classified into clusters based on Agglomerative Hierarchical Clustering (AHC). An example is given by the pavement sections in figure 1 . Figure 1.a represents the degradation of each section. By applying the AHC algorithm, the clustering process is given by the figure 1.b called dendrogram. Each cluster groups the sections degrade with the same way (figure 1.c).

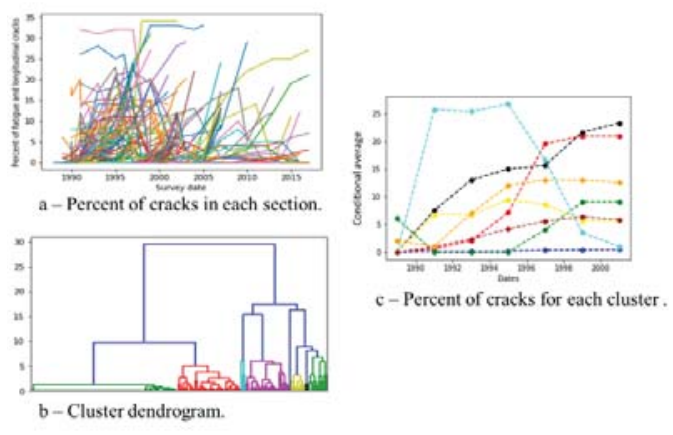

Fig. 1. Clustering of the pavement sections.

In this case, the road infrastructure is presented as a multicomponent system S composed of sub-systems S'. Each sub-system is itself a multicomponent system with 4 components (3 road markings and the pavement).

According to $W E M$ algorithm presented in $\mathrm{M}$. Redondin et al (2018) three censored lifetimes are identified. If a failure has happened before the first inspection, the data is leftcensored. If the failure has occurred between two inspections, then the data is interval censored. In case the failure has not been observed, the data is right censored. By applying the $W E M$ algorithm on each cluster, the parameter of Weibull distribution and the lifetime are estimated.

\section{Grouping maintenance strategy}


The grouping maintenance strategy proposed in this paper is an improvement or an extension of the work presented in I. Najeh et al (2020). Considering the road infrastructure as a serial system $S$ with $N$ components $(N=4$ ou 3$)$. As mentioned in the previous section, a maintenance strategy is defined for each component $i$ with $i \in$ $1, \ldots, N$,considering an age-based maintenance policy. Each component is preventively replaced after a fixed interval of time. If between two preventive maintenance the component fails, a corrective maintenance is performed to restore the component "as good as new".

\subsection{Preventive age-based replacement}

Each component $i$ of the system $S$ is associated to the Weibull distribution $W\left(\alpha_{i}, \beta_{i}\right)$. The figure 2 presents the replacement strategy.

As illustrated in the Figure 2, for each component $i$ the maintenance is performed every $T_{i}^{*}$ time unit. The preventive maintenance cost for the component $i$ is $C_{p}^{i}$. If the component is replaced between two replacement campaigns, the corrective maintenance cost is $C_{\mathrm{c}}^{i}$. The maintenance is then reset, and the next maintenance is schedule after $T_{i}^{*}$ time unit.

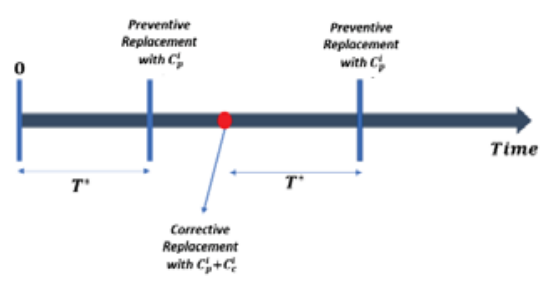

Fig. 2. Age-based replacement strategy.

To identify the optimal replacement age, the renew theorem identify the function (1) as the mean maintenance cost by time unit over an infinite horizon Rausand and Hoyland (2004). $F_{i}$ and $R_{i}$ are respectively the cumulative and reliability functions associated to $W\left(\alpha_{i}, \beta_{i}\right)$.

$$
C_{i}(t)=\frac{C_{p}^{i}+C_{c}^{i} F_{i}(t)}{\int_{0}^{t} R_{i}(x) d x}
$$

Finally the optimum replacement step is defined by minimizing the eq(2).

$$
T_{i}^{*}=\left\{\tau / C_{-} i(\tau)=\min _{t>0} C_{-} i(t)\right\}
$$

\subsection{Planning horizon}

To identify the optimal time to perform the maintenance activities simultaneously, a planning $(H Z)$ horizon must be defined. It begins at $T_{\text {Begin }}$ and ends $T_{E n d}$. H. Vu et al (2014) proposed to identify the $T_{\text {End }}$ as follows:

$$
T_{E n d}=\min _{i=1, \ldots, N} t_{i}^{1}
$$

Where $t_{i}^{1}$ represents the date of the first maintenance action of the component $i$. In this work, we suppose that all the components have just been replaced, thus $\operatorname{age}_{i}^{0}=0 \forall i \in$ $1, \ldots, N$, where $a g e_{i}^{0}$ represents the age of the component $i$ at $T_{\text {Begin }}\left(T_{\text {Begin }}=0\right)$. For the road infrastructure, the grouping planning horizon is defined by $H Z=[0,360]$ where 360 mounths represents 30 years which is the life span of the pavement.

\subsection{Optimization of the solution}

To select the best solution, a Genetic Algorithm is used in this work. This algorithm is based on principles of natural selection and genetics (A. S. Fraser (1957), H. J. Bremermann (1958), Holland (1992)).

Genetic algorithm requires a coding of the solution in the form of a table $X$ in the size is equal to the number of maintenance actions scheduled during the planning horizon. Many papers propose a binary coding (H. Yaohua, and C. W. Hui (2010), A.H. Wright (1991)). In this work, the coding method presented by H.C Vu (2014) is used. The vector $\mathrm{X}$ is structured as:

$$
i^{j} \in G^{k} \rightarrow X\left(i^{j}\right)=k, k \in[1, \ldots,(N-1)]
$$

Where $i^{j}$ is the $j^{t h}$ maintenance action of the component $i$ and $k$ is the number of the group $\mathrm{G}$ that contains the action $i^{j}$. To ensure that there will be at least two maintenance actions performed simultaneously, the maximum number of groups is $(N-1)$. The genetic algorithm process starts by introducing an initial generation randomly generated.

To illustrate the coding method, an example of candidate solution is given by figure 3. Let's $\mathrm{S}$ be a multicomponent system with 4 components and 6 maintenance actions scheduled in the planning horizon. This solution proposes to group maintenance action into 4 groups.

\begin{tabular}{|c|c|c|c|c|c|}
$1^{1}$ & $1^{2}$ & $2^{1}$ & $3^{1}$ & $3^{2}$ & $4^{1}$ \\
\hline 1 & 2 & 2 & 3 & 4 & 4 \\
\hline
\end{tabular}

Fig. 3. Example of candidate solution.

So that a solution is "acceptable" if certain constraints are respected. If two maintenance actions of the same component belong to the same group, one of them is randomly moved to another group. If the $(j+1)^{\text {th }}$ of the 
component $i$ belongs to the group $\mathrm{k}$ while the $j^{\text {th }}$ occurrence belongs to the group $l$ with $k<l$, the two actions are reversed. Thus, before launching the different steps of the genetic algorithm, a filter to correct the given solutions is applied. Afterwards, we move on to the selection phase. In this step, Roulette Wheel selection is used. A selection likelihood is associated to each solution proportionally to its performance. The selected solutions (the parents) are used to create a new one by crossing the parents. The crossover point is randomly chosen. To generate new solutions, an action maintenance is moved from a group to another. To ensure that the candidate solution is "acceptable", the filter applied before the selection phase is practiced. The schema presented by figure 4 summaries the phase of the genetic algorithm.

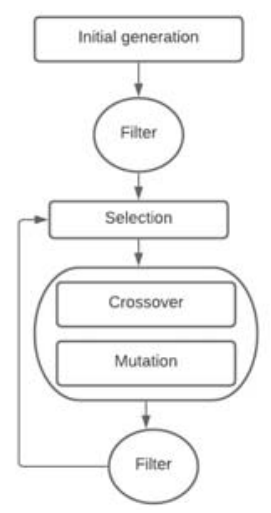

Fig. 4 Genetic algorithm process.

\subsection{Mathematical formulation of the economic profit}

To identify the best solution, we calculate the economic profit associated. It is divided into 3 parts. For each group $G^{k}$ the economic profit is presented as:

$$
E P\left(G^{k}\right)=U_{G^{k}}-\Delta H_{G^{k}}^{1}+\Delta H_{G^{k}}^{2}
$$

$U_{G^{k}}$ is the setup cost. When the maintenance actions are simultaneously performed, only one setup cost is required. Thus $U_{G^{k}}=\left[\operatorname{Card}\left(G^{k}\right)-1\right] . S_{p}$, where $\operatorname{Card}\left(G^{k}\right)$ is the number of preventive maintenance actions of the group $\mathrm{k}$ and $S_{p}$ is the setup cost.

When maintenance actions are grouped, their maintenance execution times are changed. As shown in the figure (5), $t^{\prime}{ }^{j}=t_{i^{j}}+\Delta t_{i^{j}}$ where $\Delta t_{i^{j}}>-x_{i}{ }^{*}$. The change of execution time leads to the penalty cost. For each preventive maintenance action $i^{j}$ belonging to the group $\mathrm{k}$, the penalty cost is defined as:

$$
\begin{aligned}
h_{i}\left(\Delta t_{i^{j}}\right)=E[ & {\left[M C_{i}\left(x_{i}^{*}+\Delta t_{i^{j}}\right)\right]-E\left[M C_{i}\left(x_{i}^{*}\right)\right] } \\
& -\Delta t_{i^{j}} \cdot C A^{*}
\end{aligned}
$$

Where $M C_{i}(x)=C_{i}^{c} \cdot\left(\frac{x}{\alpha}\right)^{\beta}$ the total corrective maintenance cost in the interval time $[0, x]$ for the component $i$ and $C A^{*}=\Delta t_{i} j \cdot \frac{C_{i}^{*} \beta_{i}}{x_{i}^{*}\left(\beta_{i}-1\right)}$ the average asymptotic cost. The penalty cost of the group $G^{k}$ is computed as:

$$
\Delta \mathrm{H}_{\mathrm{G}^{\mathrm{k}}}^{1}=\sum_{i^{j} \in G^{k}} h\left(\Delta t_{i^{j}}\right)
$$

The optimal execution time of given group $G^{k}$ is defined by minimizing the equation (7).

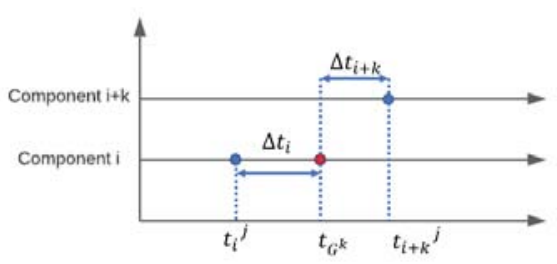

Fig 5: Change of maintenance dates.

The third part of the economic profit is additional gain due to the structure $\Delta \mathrm{H}_{\mathrm{G}^{\mathrm{k}}}^{2}$ given by the equation ().

$$
\Delta H_{G^{k}}^{2}=\pi_{G^{k}} \cdot\left(C_{\overline{G^{k}}}-C_{G^{k}}\right)
$$

Where $C_{\overline{G^{k}}}$ is the total planned shutdown cost if the maintenance activities are performed separately, and $C_{G^{k}}$ is the planned shutdown cost when maintenance activities are performed simultaneously. While $\pi_{i}$ is an indicator function characterized by:

$$
\pi_{i}=\left\{\begin{array}{c}
1 \quad \text { if the component } i \text { is critical } \\
0 \text { if the component } i \text { is not }
\end{array}\right.
$$

A component is said critical if its shutdown leads to the shutdown of all the system and non-critical if the system is still in functioning albeit the component fails.

The total economic profit of a candidate solution SGM is given by:

$$
T E P(S G)=\sum_{G^{k} \in S G M} E P\left(G^{k}\right)
$$

In order to identify the optimal grouping maintenance for the system, the equation (10) is maximizing.

\subsection{Updating the maintenance planning in dynamic contexts}


To provide the grouping maintenance strategy, a dynamic context is included. This dynamic context refers to the changes at components level. Those changes may be due the components characteristics or related maintenance costs with changes of maintenance costs or investment costs due to the price fluctuations. The main idea of the proposed model is to dynamically optimize the maintenance decisions of multi-component systems. This approach is built on monitoring information on components and maintenance operations must be dynamically grouped.

Keeping the same periodicity of inspection for the road marking of the French National Road 4, the marking lines are inspected. The level of retro reflection $R L$ at the time of inspection (time of observation) $T_{o b s}$ in given by the equation (11).

$$
\begin{array}{r}
R L^{j}\left(T_{o b s}\right)=R L_{W}^{j}\left(T_{o b s}\right)+m^{j}\left(T_{o b s}\right) \cdot\left(150-R L_{W}^{j}\left(T_{o b s}\right)\right) \\
m^{j}\left(T_{o b s}\right)=1-\frac{1}{1+\left(\frac{T_{o b s}}{T_{m_{j}}}\right)^{\alpha j}}
\end{array}
$$

Where $R L^{j}\left(T_{o b s}\right)$ is the retro reflection level of the line $\mathrm{j}$ at $T_{o b s}, R L_{W}^{j}\left(T_{o b s}\right)$ the retroreflection level of the line $\mathrm{j}$ given by the parameters of the associated Weibull distribution, $m^{j}\left(T_{o b s}\right)$ is degradation coefficient which varies between 0 and 1 and given by the equation (12) with $T_{m_{j}}$ is the instant when the retro reflection level reach the critical threshold $150 \mathrm{mcd} / \mathrm{m}^{2} / \mathrm{lx}$.

Once the level of retro reflection is estimated, the individual maintenance plan is updated: a new maintenance date is therefore defined. Depending on this new maintenance dates, we restart the grouping to optimize the grouping maintenance. To facilitate the understanding of the developments of the dynamic grouping strategy, the figure 5 illustrates the approach.

To calculate the economic profit in the case of dynamic grouping of maintenance actions $\left(T E P_{D}(S G)\right)$, it is necessary to take into account the inspection cost $C_{i n s}$. In this incident, the economic profit is given by equation (13).

$$
T E P_{D}(S G)=T E P(S G)-n * C_{\text {ins }}
$$

Where $n$ is the number of inspection scheduled during the planning horizon.

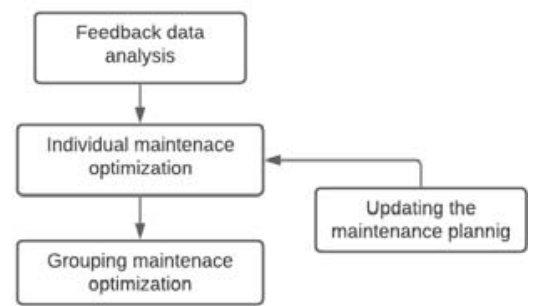

Fig. 6 The phases of dynamic grouping approach.

To illustrate the approach described in this work, an example is given by the first cluster. It is a cluster with 4 components. The following table summarizes the Weibull distribution parameters $(\alpha, \beta)$ associated to each component and the individual maintenance path. 11)

\begin{tabular}{|c|c|c|c|}
\hline Components & $\alpha$ & $\beta$ & $T_{i}^{*}$ \\
\hline Pavement & 128.7 & 7.25 & 132 \\
\hline Median strip line & 21.84 & 2.01 & 14 \\
\hline Emergency line & 23.8 & 2.02 & 18 \\
\hline Skip centre line & 30.34 & 6.64 & 40 \\
\hline
\end{tabular}

Table 1. Result of application of the EM algorithm and the maintenance strategy

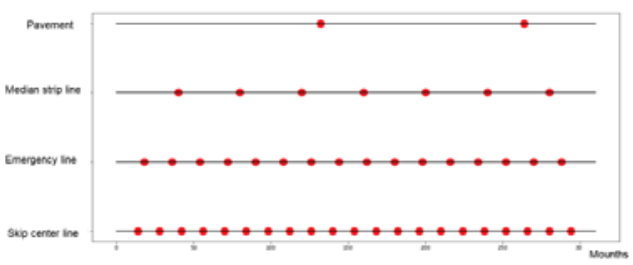

Fig. 7 Maintenance actions scheduled during the planning horizon.

Figure 7 shows all maintenance actions scheduled during the planning horizon and Figure 8 shows the result of applying the static grouping maintenance. The maintenance activities are at least grouped by two. Using this policy, the economic profit is about $10.3 \%$.

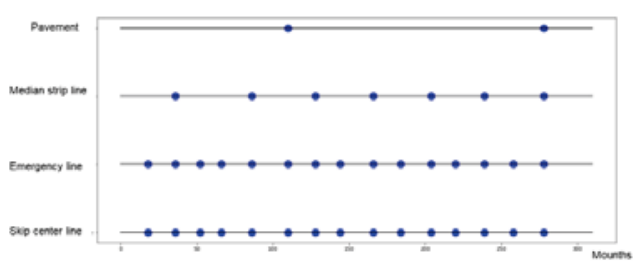

Fig. 8 Static grouping strategy.

By applying the dynamic grouping strategy presented in this work, the optimal grouping policy is given by the figure 9 . 


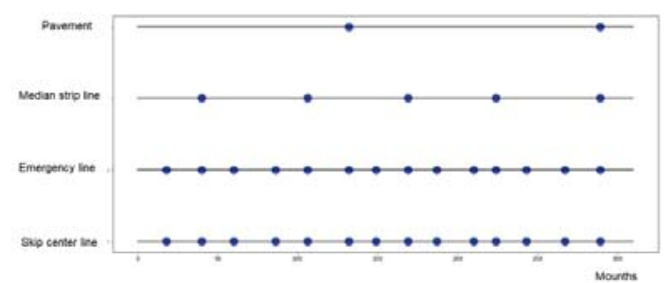

Fig. 9 Dynamic grouping strategy.

As illustrated in the previous figure, the maintenance dates actions are shifted and therefore others are deleted. Knowing that the maintenance cost is very high compared to the inspection cost, the dynamic policy helps to reduce costs and insure a good quality of the road infrastructure. In this case, the economic profit is $13.47 \%$.

\section{Conclusion and perspectives}

This paper presents a dynamic grouping maintenance actions for road infrastructure. It aims to optimize the availability of the road infrastructure to better accommodate the autonomous vehicle. Thus, efficient maintenance should be taken into account. It assumed that the infrastructure is a serial system with 4 components (3 road markings and pavement). The proposed strategy is applied on the feedback data of both French National Road 4 (for road marking) and American database Long Term Pavement Performance (LTPP) (for the pavement).

The road infrastructure considered in this paper is periodically inspected. In this case the data is censored (left censored, interval censored or right censored). To simplify the study, the data is classified into clusters that group together the section that deteriorate with the same way. The WEM algorithm is applied to estimate the failure time and parameters associated to the Weibull distribution. Based on those parameters and the maintenance costs (corrective and preventive) of each component, the optimal replacement path is defined. To minimize the maintenance cost, the grouping maintenance strategy is applied. This strategy is stationary. To improve this policy, a dynamic context is included. It is about including short time information. In this case, the road markings are periodically inspected (once a year). The result of the inspection helps to update the individual maintenance plan. According to this new maintenance dates, the grouping plan is relaunched to optimize the maintenance plan as it goes along. The dynamic maintenance policy is an approach that guarantees to have a road infrastructure in good condition and to minimize the preventive maintenance costs.
Future works aim to vary the periodicity or the frequency of inspection to study the impact of this inspection path on the changes of the grouping maintenance policy. Another approach can be envisaged by imagining inspections made by the cameras of the autonomous vehicle (or with vehicle equipped with camera). This method can provide more information about the condition of the road infrastructure.

\section{Acknowledgement}

VEDECOM and IFSTTAR thank the DIR EST and CEREMA for inspections data of the marking lines of the French National Road 4. Thanks to the Federal Highway Administration for making the LTPP database accessible.

\section{References}

Vu, C., Do, P., Barros, A., and C. Bérenguer. (2014), Maintenance grouping strategy for multi component systems with dynamic contexts. Reliability Engineering \& System Safety 132 233-249.

Bouvard, K., S. Artus, C. Bérenguer, and V. Cocquempot. (2011), Condition-based dynamic maintenance operations planning \& grouping. Application to commercial heavy vehicles. Reliability Engineering \& System Safety, 96(6), 601-610.

Nguyen, H. S. H., P. Do Van, B. Iung, and H. C. Vu. (2017). A dynamic grouping maintenance strategy for geographically dispersed production systems. In 10th International Conference on Mathematical Methods in Reliability, MMR.

Najeh, I., L. Bouillaut, D. Daucher and M. Redondin. (2020). Maintenance strategy for the road for the Autonomous Vehicle, ESREL 2020-PSAM 15, 30 ${ }^{\text {th }}$ European Safety and Reliability Conference and the $15^{\text {th }}$ Probabilistic Safety Assessment and Management Conference.

Do Van, P., A. Barros, C. Bérenguer, K. Bouvard, F. Brissaud. (2013). Dynamic grouping maintenance with time limited opportunities. Reliability Engineering \& System Safety, 120, 51-59.

Fraser, A. S. (1957). Simulation of genetic systems by automatic digital computers I. Introduction. Australian Journal of Biological Sciences, 10(4), 484-491.

Bremermann. H. J. (1958). The evolution of intelligence: The nervous system as a model of its environment. University of Washington, Department of Mathematics.

Holland, J. H. (1992). Adaptation in natural and artificial systems: an introductory analysis with applications to biology, control, and artificial intelligence. MIT press.

Yaohua, H., and C. W. Hui (2010). "A binary coding genetic algorithm for multi-purpose process scheduling: A case study." Chemical engineering science 65.16.

Wright, A.H. (1991). "Genetic algorithms for real parameter optimization." Foundations of genetic algorithms. Vol. 1. Elsevier.

Wildeman, R.E, R. Dekker, and A. C. J. M. Smit. (1997)"A dynamic policy for grouping maintenance activities." European Journal of Operational Research 99.3 530-551

Revilloud, M, D. Gruyer, \& M. C. Rahal (2016, May). "A new multi-agent approach for lane detection and tracking." In 2016 IEEE International Conference on Robotics and Automation (ICRA) (pp. 3147-3153). IEEE. 
Redondin, M., Faul, N., Bouillaut, L., Same, A., \& Daucher, D. (2018, June). Alternative Weibull analysis for road markings: an EM approach. In ESREL2018-European Safety and Reliability Conference (p. 7p).

Rausand, M., \& A. Hoyland. (2004). Component importance. System Reliability Theory: Models, Statistical Methods, and Applications. 